\title{
Congenital Adhesion Band Presenting as Intestinal Perforation in an Extremely Low Birth Weight Infant
}

\author{
Juan Guillen, MD ${ }^{1}$ Stacey Ramey, MD $^{1}$ Prabhu Satya Parimi, MD, MBA, CPE ${ }^{1}$ \\ ${ }^{1}$ Department of Pediatrics, Neonatology, Metro Health Medical \\ Center, Cleveland, Ohio \\ Am J Perinatol Rep 2021;11:e1-e4. \\ Address for correspondence Prabhu S. Parimi, MD, MBA, CPE, \\ Department of Pediatrics, Neonatology, Metro Health Medical Center, \\ 2500 MetroHealth Drive, Cleveland, OH 44109-1998 \\ (e-mail: pparimi@metrohealth.org).
}

\begin{abstract}
Keywords

- perforation

- congenital band

- premature infant

- laparotomy

A $23^{1 / 7}$-week-old and 560-g-weighing premature male infant was evaluated on day of life 33 for increased frequency of bradycardias, bilious residual, and an increase in abdominal girth. Physical examination was notable for distended and mild tender abdomen. Investigations revealed pneumoperitoneum with dilated bowel loops and a normal acid-base balance. An urgent exploratory laparotomy demonstrated isolated jejunal perforation with an adhesive band extending from the omentum to the base of the mesentery. A segmental jejunal resection followed by an end-to-end anastomosis was performed. The diagnosis of intestinal perforation was inconsistent with focal spontaneous intestinal perforation and necrotizing enterocolitis. Decision to perform exploratory laparotomy led to diagnosis of congenital adhesion band, a rare clinical condition, and the patient had a favorable outcome. This premature infant made an excellent recovery and the upper gastrointestinal $(\mathrm{Gl})$ study demonstrated that the anastomotic site was intact. He is currently tolerating advancing enteral feeds.
\end{abstract}

Careful consideration of the clinical history with a well thought out surgical plan offers the best possible care and outcome for premature infants with intestinal perforation. This report illustrates prompt diagnosis of intestinal perforation and the critical surgical decision-making process in a premature infant with yet unreported congenital adhesion band leading to ischemia and isolated perforation of the jejunum. Provision of crucial pieces of clinical data and collaboration aids surgeons in adopting a precision-based surgical management approach for patients with intestinal perforation.

Focal spontaneous intestinal perforation (SIP) and necrotizing enterocolitis (NEC) are the two most common yet rare causes of perforation occurring in approximately $1 \%$ of premature infants ${ }^{1}$; however, congenital adhesion band presenting as intestinal perforation in a preterm infant has not been reported. Congenital adhesion band is a rare cause of small bowel obstruction across all age groups with an

received

September 18, 2020

accepted

November 4, 2020
DOI https://doi.org/

$10.1055 / \mathrm{s}-0040-1722729$. ISSN 2157-6998. incidence ranging between 3.3 to $28 \%{ }^{2,3}$ Only three cases of congenital intestinal adhesion band are reported in neonates thus far with the band from the mesenteric root compressing ileum in two patients and jejunum in one patient. All neonatal patients had undergone segmental resection. ${ }^{4}$

We report a case of congenital adhesion band involving the jejunum in a premature infant, $23^{1 / 7}$ weeks of gestational age, presenting with intestinal perforation. The congenital band was present $30 \mathrm{~cm}$ from the ligament of Treitz and terminating at the site of intestinal perforation with intraoperative findings of volvulus. Prompt diagnosis of intestinal perforation, but more importantly the decision to perform exploratory laparotomy, led to intraoperative and histological diagnosis. This case amplifies the importance of taking a precision-based approach to a surgical emergency in an extremely low birth weight infant which was crucial to preserve the intestine with excellent postoperative outcome.
(C) 2021. The Author(s).

This is an open access article published by Thieme under the terms of the Creative Commons Attribution-NonDerivative-NonCommercial-License, permitting copying and reproduction so long as the original work is given appropriate credit. Contents may not be used for commercial purposes, or adapted, remixed, transformed or built upon. (https://creativecommons.org/ licenses/by-nc-nd/4.0/)

Thieme Medical Publishers, Inc., 333 Seventh Avenue, 18th Floor,

New York, NY 10001, USA 


\section{Case Presentation}

An extreme low birth weight infant (ELBW) with $23^{1 / 7}$ weeks of gestational age and birth weight of $560 \mathrm{~g}$ was delivered by an emergency cesarean section due to nonreassuring fetal heart tracings with an Apgar's score of 4, 6, 7 at 1, 5, and 10 minutes, respectively. Pregnancy was complicated by premature prolonged rupture of membranes and multiple Escherichia coli urinary tract infections. A rescue cerclage was placed due to cervical insufficiency and a course of indomethacin was given to avert preterm labor. As mother continued to experience irregular and persistent contractions, she was given betamethasone. Baby was intubated in the delivery room, received surfactant, and was placed on high-frequency oscillator due to the need for high mean airway pressure. Physical examination at birth was unremarkable and consistent with gestational age. His hospital course was complicated by hypoxemic respiratory failure necessitating the use of high-frequency oscillator and inhaled nitric oxide, hypotension requiring dopamine, ampicillin, and cefotaxime for 7 days due to presumed sepsis and leukemoid reaction. As he was predicted to have moderate-to-severe bronchopulmonary dysplasia based on the NICHD (National Institute of Child Care and Development) calculator, an 18-day tapering dose of hydrocortisone was started per the unit protocol. He was transitioned to the conventional ventilator on day of life 21 and was started on trophic feeds using donor human milk. On day of life 25 , he was on Vapotherm $4 \mathrm{~L}$ in room air, receiving $0.5 \mathrm{~mL}$ of donor human milk via nasogastric tube every 2 hours and was on parenteral nutrition. He had been on intravenous (IV) caffeine since birth with infrequent nonsignificant bradycardias. At the chronological age of 33 days at $27^{6 / 7}$ weeks of postconceptual age, he developed an increased frequency of bradycardias requiring intervention, bilious gastric aspirate, and increase in abdominal girth. Physical examination was remarkable for abdominal distension and mild tenderness.

\section{Investigations}

An abdominal X-ray obtained due to increased frequency of bradycardias, bilious aspirate, and abdominal distension demonstrated a large pneumoperitoneum and dilated bowel loops (-Fig. 1). The arterial blood gas was unremarkable with

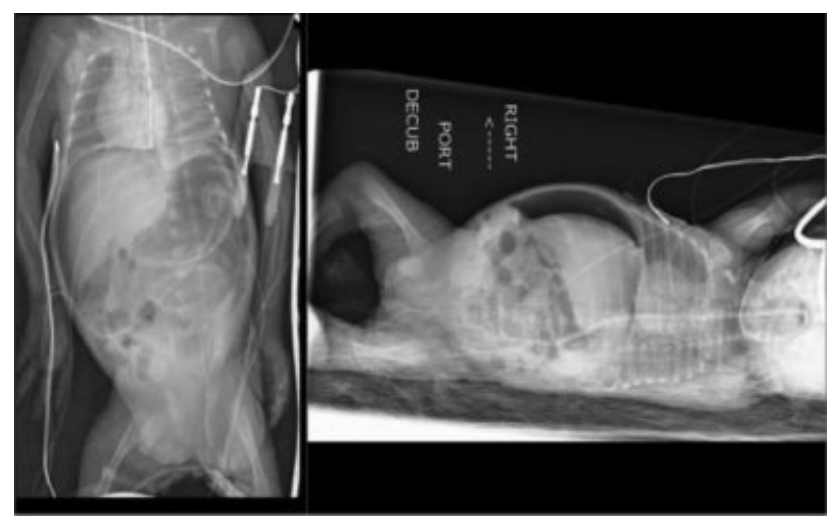

Fig. 1 AP and left lateral decubitus abdominal radiograph demonstrating pneumoperitoneum. AP, anteroposterior.

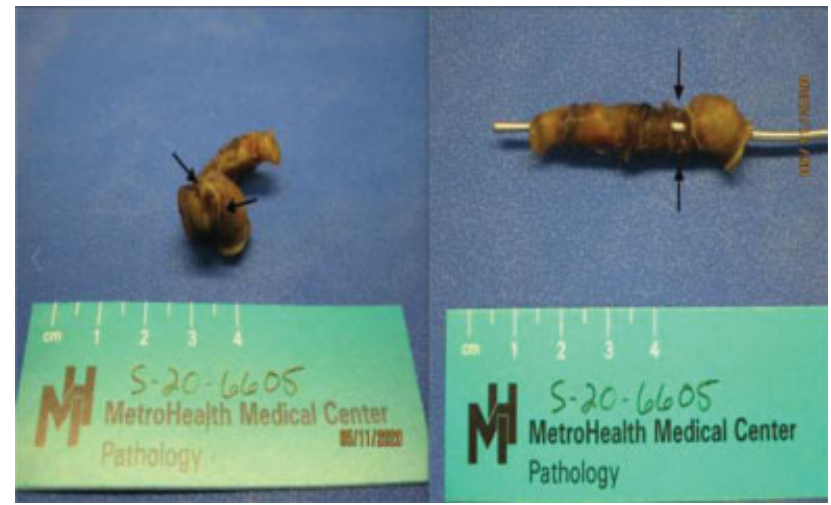

Fig. 2 Gross intestinal specimen demonstrating intestinal band and intestinal perforation.

normal pH and without base deficit. The complete blood count and C-reactive protein (CRP) were unremarkable. The patient was started on vancomycin and piperacillin-tazobactam for gram-positive, gram-negative, and anaerobic coverage given the presence of a central line in the setting of an intestinal perforation. The infant remained hemodynamically stable and was electively intubated for surgical intervention. After careful review of his clinical course since birth, age, and cardiorespiratory stability at the time of diagnosis, the surgical team decided to perform exploratory laparotomy. This revealed volvulus of the jejunum and an isolated perforation of $30 \mathrm{~cm}$ from the ligament of Treitz secondary to an adhesive band extending from the omentum to the base of the mesentery (-Fig. 2). The diagnosis of congenital adhesion band was subsequently confirmed by histopathological examination of the specimen.

\section{Differential Diagnosis}

Our differential diagnosis included SIP and stage-3 NEC, although both clinical conditions seemed unlikely in our patient. Focal SIP typically occurs early in life, around 7 days, in preterm infants with similar characteristics and risk profile as our patient. However, risk factors for SIP, such as the absence of low Apgar's scores, hemodynamically significant patent ductus arteriosus, indomethacin administration, and the use of early postnatal steroids, were not present in our patient making it less likely. Moreover, abdominal X-ray findings were not inconsistent with SIP; our patient had dilated bowel loops instead of typical gasless abdomen. Stage-3 NEC appeared unlikely given that our patient was never advanced beyond trophic feeds, previously had normal abdominal examination, and X-ray with an acute change in the clinical status and development of pneumoperitoneum. This thought process led to exploratory laparotomy being performed instead of insertion of peritoneal drain at the bedside.

\section{Treatment}

The exploratory laparotomy revealed a $50 \%$ circumferential perforation which measured to be $30 \mathrm{~cm}$ from the ligament of Treitz. Contained within the perforation, an adhesive band was identified. The adhesive band extended from the base of 
the mesentery to the omentum, with incarcerated loops of bowel underneath it, and volvulus of intestine. Several loops of adjacent small bowel had segmental bruising. After releasing the band, segmental resection was performed followed by end-to-end anastomosis.

\section{Outcome and Follow-up}

Postoperatively, the infant remained on minimal ventilatory support for 14 days and was extubated to noninvasive respiratory support without the need for supplemental oxygen. An upper gastrointestinal (GI) study was performed on postoperative day 7 which was unremarkable without evidence of leakage at the anastomotic site. He was treated with piperacillin-tazobactam for 10 days. Currently he is 63 days old, $32^{1 / 7}$ weeks of postconceptional age, and weighs $1,535 \mathrm{~g}$. The infant is on minimal noninvasive respiratory support and tolerating advancing enteral feeds.

\section{Discussion}

Congenital adhesion band is a very uncommon cause of small bowel obstruction due to entrapment of the intestinal loop between the mesentery and the band. Typical presentation in the pediatric population is with small bowel obstruction, with symptoms of bilious vomiting and abdominal distention. The ilium is the most common location of obstruction. Symptoms of small bowel obstruction occurs in up to two-thirds of patients prior to 24 months of age, with only $20 \%$ of these manifesting during the neonatal period. Most patients require prompt surgical intervention to release the band with segmental bowel resection. Intraoperative finding of volvulus is most often noted in neonates. ${ }^{4,5}$ However, congenital adhesion band presenting as pneumoperitoneum in the neonatal population has not been previously reported.

Congenital adhesion bands are of embryological origin, which arise due to persistent or incomplete regression of the fetal vitelline circulation, defects in embryogenesis, as a result of intrauterine infections or may merely be a remnant of ventral mesentery. ${ }^{6}$ Identification of a congenital adhesion band in our patient at $27^{6 / 7}$ weeks of postconceptual age supports the notion that it is of embryological origin. Extension of the band from the base of the mesentery to the omentum with volvulus of the intestine led to significant focal vascular compromise, necrosis, and perforation. This was supported by the existence of the band within the perforation on gross pathology review in addition to histological evidence of loss of bowel layers and congestion with contiguous areas demonstrating viable bowel wall (-Fig. 3 ).

The case described here exemplifies the importance and adoption of precision-based surgical approach in premature infants with intestinal perforation. Scrutiny of the preceding clinical course is critical in choosing the right surgical approach of either bedside insertion of a peritoneal drain versus exploratory laparotomy. Our patient's clinical presentation was inconsistent with the diagnosis of SIP or stage-3 NEC and therefore the choice of exploratory laparotomy was necessary to make the diagnosis of congenital adhesion band.

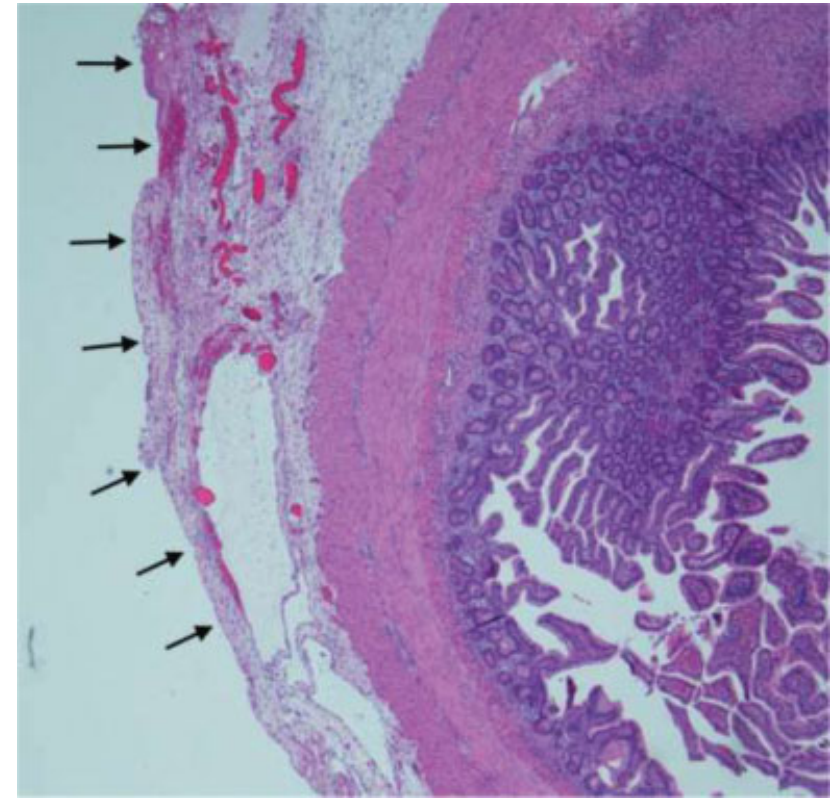

Fig. 3 Histopathological examination of the involved bowel segment showing intestinal band (indicated by black arrows).

Given the intraoperative findings in our case, we believe that careful review of the clinical course and timely surgical intervention with exploratory laparotomy resulted favorable clinical outcome in this extremely low birth weight infant.

\section{Learning Points/Take Home Messages}

1. Congenital intestinal band presenting as pneumoperitoneum in an extremely low birth weight infant has not been reported.

2. Careful review of clinical course is critical to entertain and identify other causes of intestinal perforation in premature infants

3. A collaborative approach is crucial to determine the choice of surgical intervention, bedside peritoneal drain versus exploratory laparotomy

Conflict of Interest

None declared.

\section{References}

1 Shah J, Singhal N, da Silva O, et al;Canadian Neonatal Network. Intestinal perforation in very preterm neonates: risk factors and outcomes. J Perinatol 2015;35(08):595-600

2 Weibel MA, Majno G. Peritoneal adhesions and their relation to abdominal surgery. A postmortem study. Am J Surg 1973;126 (03):345-353

3 Butt MU, Velmahos GC, Zacharias N, Alam HB, de Moya M, King DR. Adhesional small bowel obstruction in the absence of previous operations: management and outcomes. World J Surg 2009; 33(11):2368-2371

4 Yang KH, Lee TB, Lee SH, Kim SH, Cho YH, Kim HY. Congenital adhesion band causing small bowel obstruction: what's the difference in various age groups, pediatric and adult patients? BMC Surg 2016;16(01):79 
e4 Intestinal Perforation in an Extremely Low Birth Weight Infant Guillen e al

5 Erginel B, Soysal FG, Ozbey H, et al. Small bowel obstruction due to anomalous congenital bands in children. Gastroenterol Res Pract 2016;2016:7364329
6 Arung W, Meurisse M, Detry O. Pathophysiology and prevention of postoperative peritoneal adhesions. World J Gastroenterol 2011;17(41):4545-4553 